\title{
Prognostic relevance of autophagy markers LC3B and p62 in esophageal adenocarcinomas
}

\author{
Olivia Adams ${ }^{1,2}$, Bastian Dislich ${ }^{1}$, Sabina Berezowska ${ }^{1}$, Anna M. Schläfli ${ }^{1}$, Christian \\ A. Seiler ${ }^{3}$, Dino Kroell ${ }^{3}$, Mario P. Tschan ${ }^{1,2}$ and Rupert Langer ${ }^{1}$ \\ ${ }^{1}$ Institute of Pathology, University of Bern, Bern, Switzerland \\ ${ }^{2}$ Graduate School for Cellular and Biomedical Sciences, University of Bern, Bern, Switzerland \\ ${ }^{3}$ Department of Visceral Surgery and Medicine, Inselspital University Hospital Bern and University of Bern, Bern, Switzerland \\ Correspondence to: Rupert Langer, email: rupert.langer@pathology.unibe.ch \\ Keywords: esophageal adenocarcinoma, autophagy, LC3B, p62, Pathology Section \\ Received: January 05, $2016 \quad$ Accepted: May 13, 2016 \\ Published: May 26, 2016
}

\section{ABSTRACT}

Esophageal adenocarcinomas (EAC) are aggressive tumors with considerable rates of chemoresistance. Autophagy is a lysosome-dependent degradation process, characterized by the formation of vesicles called autophagosomes, and has been implicated in cancer. Protein light chain 3 B (LC3B) and p62 are associated with autophagosomal membranes and degraded. We aimed to assess the impact of basal autophagy on EAC. In EAC cell lines, an increase in LC3B and p62 was observed with increasing concentrations of the autophagy inhibitor chloroquine, which indicates functional basal autophagy. LC3B and p62 immunohistochemistry was performed on primary resected EAC. High LC3B and p62 expression was associated with earlier tumor stages $(p<0.05)$. High nuclear and cytoplasmic p62 staining were associated with a better prognosis $(p=0.006 ; p=0.028)$. Various combinations of $p 62$ expression with or without LC3B expression identified different prognostic groups. Tumors with low total p62 $(p=0.007)$ or low LC3B/low p62 expression had the worst outcome $(p=0.007 ; p=0.005)$. A combination score of dot-like/cytoplasmic $p 62$ and nuclear p62 staining was an independent prognostic parameter $(p=0.033$; HR $=0.6)$. This study highlights the potential significance of basal autophagy in EAC biology. Tumors with low LC3B and p62 expression show the most aggressive behavior and may be candidates for autophagy regulating therapeutics.

\section{INTRODUCTION}

Esophageal Adenocarcinomas (EAC), usually arising from metaplastic Barrett's esophagus (BE), are highly aggressive tumors and often locally and systemically advanced upon diagnosis. Furthermore, the incidence is increasing in developed countries [1]. Treatment for locally advanced disease has improved with refined surgical techniques and the advent of neoadjuvant chemotherapy. However, the therapeutic options for recurrent and metastatic disease are mainly limited to (radio)chemotherapy leading to an overall poor prognosis for EAC [2]. Alternative therapeutic strategies, which also may encompass targeting molecular events/regulation, would therefore be highly desirable.

Macroautophagy (hereafter referred to as autophagy) is a cellular catabolic process for the degradation and recycling of cellular components such as proteins and organelles. The process is tightly regulated and highly conserved. Under basal conditions autophagy maintains homeostasis. Autophagy is a pro-survival mechanism under cellular stresses, such as nutrient deprivation, as it frees up building blocks and energy for essential processes [3]. The dysregulation of autophagy has been implicated in many diseases including neurodegenerative diseases. In cancer autophagy has demonstrated diverging roles, being described as both pro- and anti-oncogenic. It is theorized that autophagy can have a tumor suppressor function in early stages as it degrades deleterious proteins and maintains genomic stability. However, later on autophagy can be exploited by neoplastic cells as a survival mechanism enabling progression, invasion, metastasis and 
evading cell death upon treatment [4]. Autophagy also has diverging roles with respect to tumor immune surveillance given its effect on the tumor microenvironment and function in antigen presentation $[5,6]$. Therefore the first aim of this study was to confirm functional basal autophagy in EAC cell lines. Functional basal autophagy can be defined as the continual autophagic flux under steady state culture conditions in the absence of chemo-, radio or targeted therapy. This is of relevance given the recent characterization of cancer cell lines lacking core autophagy machinery components and consequently proving unable to undergo functional basal autophagic flux $[7,8]$. While cancer cell lines lacking functional basal autophagy are rare, in light of recent discoveries, this proof of principle experiment was of import. Functional basal autophagy can be assessed via the markers LC3B and p62 in the absence and presence of pharmacological autophagy inhibition. Cytosolic LC3B-I is lipidated and incorporated into the autophagosomal membrane, forming the LC3B-II isoform. LC3B-II is subsequently degraded during autophagic flux [9]. As LC3B-II is membranebound and subsequently degraded, the accumulation of this isoform upon pharmacological autophagy inhibition is indicative of intact basal autophagy. LC3B-II can also be visualized via immunofluorescence as punctae. Similarly, the accumulation of p62, which is also associated with the autophagosomal membrane and subject to degradation, upon pharmacological autophagy inhibition is also an indicator of functional basal autophagy [9]. The second aim was to assess basal autophagy in EAC ex vivo. Immunohistochemical LC3B and p62 dot-like staining is considered to be indicative of autophagosomes and may therefore be used as markers in patient tissue. Using a previously established staining and scoring protocol [10] LC3B and p62 immunohistochemistry was performed on next generation tissue microarrays (ngTMAs) of formalin fixed paraffin embedded (FFPE) samples of primary resected EAC, Barrett's mucosa, adjacent nonneoplastic esophageal mucosa, adjacent non-neoplastic gastric mucosa, EAC lymph node metastasis and EAC distant metastasis and the results were then correlated with clinicopathological parameters.

\section{RESULTS}

\section{EAC exhibits intact basal autophagy in vitro}

To access functional basal autophagy in EAC in vitro the autophagy markers LC3B and p62 were assessed via immunoblotting and immunofluorescence upon pharmacological autophagy inhibition with chloroquine. EAC cell lines OE19 and OE33 were treated with chloroquine (CQ) for 48 hours followed by immunoblotting for LC3B and p62, with total protein was visualized as loading control. A dose-dependent increase of both proteins was observed with increasing concentrations of $\mathrm{CQ}$, which is indicative of functional basal autophagy (Figure 1). This result was verified by immunofluorescence staining in both cell lines as a dosedependent increase in LC3B-II punctae with increasing concentrations of CQ was observed, confirming functional basal autophagy (Figure 2).

\section{Clinicopathological and prognostic implications of autophagy markers in EAC tissue}

Median age of the patient cohort was 69 years (ranging from 32 to 89 years). Male/female ratio was 100/16. The pT category (according to UICC 2009 [11]) was as follows: pT1 - 35 cases $(30.2 \%)$, pT2 - 11 cases $(9.5 \%)$, pT3 - 67 cases $(57.8 \%)$ and pT4 3 cases (2.6\%). Lymph node metastases were absent in 56 cases $(48.3 \%)$ and present in 60 cases $(51.7 \%)$. Distant metastases at the time of surgery were present in 5 cases $(4.3 \%)$. Lymphatic invasion was present in 78 cases $(67.2 \%)$, venous invasion in 29 cases $(25 \%)$ and perineural invasion present in 45 cases $(38.8 \%)$. Tumor grading was classified as G1 (well differentiated) in 18 cases $(15.4 \%), \mathrm{G} 2$ (moderately differentiated) in 50 cases (43.1\%) and G3 (poorly differentiated) in 48 cases $(41.4 \%)$. Intratumoral inflammation was low in 55 cases (47.4\%) and high in 61 cases (52.6\%). Complete tumor resection (R0) was achieved in 109 cases (93.9\%). The median overall survival, which was calculated from the day of surgical resection until last contact or death, was 28 months (ranging from 0 to 208 months). Patient data is summarized in Table 1.

In primary resected EAC and primary resected nondysplastic and dysplastic Barrett's mucosa we observed LC3B (Figure 3) and p62 dot-like staining patterns ranging from absent (score 0 ) to strong (score 3 ) with detection of abundant immunoreactive dots. Similarly a range of p62 cytoplasmic staining was observed and both negative and positive p62 nuclear staining was reported (Figure 4). LC3B and p62 dot-like immunostaining showed a positive association $(p=0.020)$ in primary resected EAC tumors. A positive association between individual p62 dot-like and p62 cytoplasmic staining scores was observed $(p=0.005)$. See Supplemental material for all correlations between individual staining scores (Supplementary Table 1.1 to Supplementary Table 2.3).

Staining patterns were homogenous for LC3B and all scoring categories of p62 across the tumors. Intratumoral heterogeneity, defined as more than one of the six tumor punches (three taken from tumor center and three taken from tumor periphery) showing discrepancies greater than 1 scoring point, was in the explicit minority. 
Table 1: Summary of patient data

\begin{tabular}{|c|c|c|c|}
\hline Parameter & Category & Total & Percentage $(\%)$ \\
\hline Median age & - & 69 years & - \\
\hline Sex & \begin{tabular}{|l} 
Male \\
Female
\end{tabular} & $\begin{array}{l}100 \\
16 \\
\end{array}$ & $\begin{array}{l}86.2 \\
13.8 \\
\end{array}$ \\
\hline pT category $(\mathrm{T})$ & $\begin{array}{l}\text { pT1 } \\
\text { pT2 } \\
\text { pT3 } \\
\text { pT4 }\end{array}$ & \begin{tabular}{|l|}
35 \\
11 \\
67 \\
3 \\
\end{tabular} & $\begin{array}{l}30.2 \\
9.5 \\
57.8 \\
2.6 \\
\end{array}$ \\
\hline Lymph Node Metastasis (N) & $\begin{array}{l}\text { pN0 } \\
\text { pN1 } \\
\text { pN2 } \\
\text { pN3 }\end{array}$ & \begin{tabular}{|l|}
56 \\
19 \\
22 \\
19 \\
\end{tabular} & \begin{tabular}{|l|}
48.3 \\
16.4 \\
19.0 \\
16.4 \\
\end{tabular} \\
\hline Distant Metastasis (M) & $\begin{array}{l}\text { absent } \\
\text { present }\end{array}$ & $\begin{array}{l}111 \\
5 \\
\end{array}$ & $\begin{array}{l}95.7 \\
4.3 \\
\end{array}$ \\
\hline UICC Stage & \begin{tabular}{|l} 
IA \\
IB \\
IIA \\
IIB \\
IIIA \\
IIIB \\
IIIC \\
IV \\
\end{tabular} & $\begin{array}{l}32 \\
3 \\
18 \\
7 \\
16 \\
17 \\
18 \\
5 \\
\end{array}$ & $\begin{array}{l}27.4 \\
2.6 \\
15.4 \\
6.0 \\
13.8 \\
14.7 \\
15.5 \\
4.3 \\
\end{array}$ \\
\hline Lymphatic Invasion (L) & $\begin{array}{l}\text { absent } \\
\text { present }\end{array}$ & $\begin{array}{l}38 \\
78 \\
\end{array}$ & \begin{tabular}{|l|}
32.8 \\
67.2 \\
\end{tabular} \\
\hline Venous Invasion (V) & $\begin{array}{l}\text { absent } \\
\text { present }\end{array}$ & $\begin{array}{l}87 \\
29 \\
\end{array}$ & $\begin{array}{l}75.0 \\
25.0 \\
\end{array}$ \\
\hline Perineural Invasion (Pn) & $\begin{array}{l}\text { absent } \\
\text { present }\end{array}$ & $\begin{array}{l}71 \\
45 \\
\end{array}$ & \begin{tabular}{|l|}
61.2 \\
38.8 \\
\end{tabular} \\
\hline Grading $(\mathrm{G})$ & $\begin{array}{l}\text { G1 } \\
\text { G2 } \\
\text { G3 } \\
\end{array}$ & $\begin{array}{l}18 \\
50 \\
48 \\
\end{array}$ & $\begin{array}{l}15.5 \\
43.1 \\
41.4 \\
\end{array}$ \\
\hline Inflammation & $\begin{array}{l}\text { low } \\
\text { high }\end{array}$ & $\begin{array}{l}55 \\
61 \\
\end{array}$ & \begin{tabular}{|l|}
47.4 \\
52.6 \\
\end{tabular} \\
\hline Resection Status (R) & $\begin{array}{l}\text { R0 } \\
\text { R1 } \\
\end{array}$ & $\begin{array}{l}109 \\
7 \\
\end{array}$ & $\begin{array}{l}94.0 \\
6.0 \\
\end{array}$ \\
\hline
\end{tabular}

Table 2: Summary of staining patterns

\begin{tabular}{|l|l|l|}
\hline Staining Pattern & Low & High \\
\hline LC3B dots & $\begin{array}{l}97 \\
(83.6 \%)\end{array}$ & $\begin{array}{l}19 \\
(16.4 \%)\end{array}$ \\
\hline p62 dots & $\begin{array}{l}48 \\
(41.4 \%)\end{array}$ & $\begin{array}{l}68 \\
(58.6 \%)\end{array}$ \\
\hline p62 cytoplasm & $\begin{array}{l}12 \\
(10.3 \%)\end{array}$ & $\begin{array}{l}104 \\
(89.7 \%)\end{array}$ \\
\hline p62 nuclear & $\begin{array}{l}58 \\
(50.0 \%)\end{array}$ & $\begin{array}{l}58 \\
(50.0 \%)\end{array}$ \\
\hline p62 sum & $\begin{array}{l}22 \\
(19.0 \%)\end{array}$ & $\begin{array}{l}94 \\
(81.0 \%)\end{array}$ \\
\hline
\end{tabular}

The tumors showed higher amounts of LC3B dots, as well as p62 dot-like, cytoplasmic and nuclear staining, when compared to normal gastric and esophageal mucosa (ranging from $p<0.001$ to $p<0.02$ ). For LC3B there was no significant difference between primary tumor and metastases ( $p=0.301$ for lymph node and $p=0.083$ for distant metastases). For p62, higher p62 dot-like staining was observed in distant metastases compared to primary tumors $(p=0.022)$ and higher nuclear staining was observed in lymph node metastases compared to primary tumors $(p=0.021)$, whereas no difference was seen between the primary tumors and lymph node and distant metastases for cytoplasmic p62 staining $(p=0.835$ and $p$ $=0.480)$, neither for $\mathrm{p} 62$ dot-like staining regarding lymph node metastases $(p=0.777)$ and for $\mathrm{p} 62$ nuclear staining regarding distant metastases $(p=0.132)$.

For the purpose of further analysis IHC scores were categorized as either "low" or "high" based on prognostic 
Table 3: LC3B dot-like staining and clinicopathological features

\begin{tabular}{|c|c|c|c|c|c|}
\hline Parameter & Category & Total & LC3B Low & LC3B High & $p$-value \\
\hline pT category & \begin{tabular}{|l|} 
pT1 \\
pT2 \\
pT3 \\
pT4 \\
\end{tabular} & \begin{tabular}{|l|}
35 \\
11 \\
67 \\
3 \\
\end{tabular} & \begin{tabular}{|l|}
26 \\
9 \\
59 \\
3 \\
\end{tabular} & $\begin{array}{l}9 \\
2 \\
8 \\
0 \\
\end{array}$ & 0.283 \\
\hline \begin{tabular}{|ll} 
Lymph & Node \\
Metastasis & \\
\end{tabular} & $\begin{array}{l}\text { absent } \\
\text { present }\end{array}$ & $\begin{array}{l}56 \\
60\end{array}$ & $\begin{array}{l}47 \\
50\end{array}$ & $\begin{array}{l}9 \\
10\end{array}$ & 1.000 \\
\hline Distant Metastasis & \begin{tabular}{|l|} 
absent \\
present
\end{tabular} & \begin{tabular}{|l|}
111 \\
5
\end{tabular} & \begin{tabular}{|l|}
93 \\
4 \\
\end{tabular} & \begin{tabular}{|l|}
18 \\
1 \\
\end{tabular} & 1.000 \\
\hline UICC Stage & \begin{tabular}{|l|} 
I \\
II \\
III \\
IV
\end{tabular} & \begin{tabular}{|l|}
35 \\
25 \\
51 \\
5
\end{tabular} & \begin{tabular}{|l|}
26 \\
24 \\
43 \\
4
\end{tabular} & $\begin{array}{l}9 \\
1 \\
8 \\
1\end{array}$ & 0.165 \\
\hline $\begin{array}{l}\text { Lymphatic } \\
\text { Invasion }\end{array}$ & $\begin{array}{l}\text { absent } \\
\text { present }\end{array}$ & \begin{tabular}{|l|}
38 \\
78
\end{tabular} & $\begin{array}{l}31 \\
66\end{array}$ & $\begin{array}{l}7 \\
12\end{array}$ & 0.790 \\
\hline Venous Invasion & $\begin{array}{l}\text { absent } \\
\text { present }\end{array}$ & $\begin{array}{l}87 \\
29 \\
\end{array}$ & $\begin{array}{l}73 \\
24 \\
\end{array}$ & $\begin{array}{ll}14 \\
5\end{array}$ & 1.000 \\
\hline Perineural Invasion & \begin{tabular}{|l|} 
absent \\
present
\end{tabular} & $\begin{array}{l}71 \\
45 \\
\end{array}$ & \begin{tabular}{|l}
57 \\
40 \\
\end{tabular} & \begin{tabular}{|l|}
14 \\
5 \\
\end{tabular} & 0.305 \\
\hline Grading & $\begin{array}{l}\text { G1 } \\
\text { G2 } \\
\text { G3 } \\
\end{array}$ & \begin{tabular}{|l|}
18 \\
50 \\
48 \\
\end{tabular} & $\begin{array}{l}12 \\
42 \\
43 \\
\end{array}$ & \begin{tabular}{|l|}
6 \\
8 \\
5 \\
\end{tabular} & 0.158 \\
\hline Inflammation & $\begin{array}{l}\text { low } \\
\text { high }\end{array}$ & \begin{tabular}{|l}
55 \\
61 \\
\end{tabular} & $\begin{array}{l}47 \\
50 \\
\end{array}$ & \begin{tabular}{|l|}
8 \\
11 \\
\end{tabular} & 0.802 \\
\hline Resection Status & $\begin{array}{l}\text { R0 } \\
\text { R1 }\end{array}$ & $\begin{array}{l}109 \\
7 \\
\end{array}$ & \begin{tabular}{|l|}
90 \\
7 \\
\end{tabular} & \begin{tabular}{|l|}
19 \\
0 \\
\end{tabular} & 0.597 \\
\hline
\end{tabular}

impact as outlined in the Materials and Methods section. Categories for low and high for each staining is summarized in Table 2. Low/high groupings of p62 dots and cytoplasmic staining showed a statistically significant correlation $(p=0.026)$. For correlations of all low/high p62 groups please see Supplementary Table 3.1 to 3.3. The association of low and high groups of LC3B dot-like, p62 dot-like, p62 cytoplasmic, p62 nuclear and p62 sum scores with clinicopathological features $\mathrm{pT}$ category, presence of lymph node or distant metastases, clinical stage, lymphatic invasion, venous invasion, perineural invasaion, grading, inflammation and resection status was assessed. LC3B dot-like staining did not significantly correlate with any of those clinicopathological features (Table 3). In contrast, low p62 nuclear staining correlated with lymph node metastases $(p=0.015)$, as well as with higher grade ( $p=$ 0.042 ; Table 4). Strikingly, no stage I tumors with low p62 cytoplasmic expression were observed ( $p=0.021$; Table 4). In line with this finding, fewer stage I tumors had low p62 nuclear expression ( $p=0.042$; Table 4$)$. Similarly only two stage I tumors had a low p62 sum score $(p=0.017$; Table 5). Taken together these results indicated that low levels of p62 correlate with a more aggressive phenotype (Table 4; Table 5).

Low/high groupings of staining patterns versus overall survival were investigated using univariate analysis. Low p62 cytoplasmic staining, p62 nuclear staining and p62 sum score all significantly correlated with a worse overall survival with $p$-values equaling 0.028 ,
0.006 and 0.007 respectively (and in trend p62 dot-like staining $p=0.078$; Figure 5). Low LC3B was associated with worse outcome as well, but this was not statistically significant ( $p=0.335$; see Supplementary Figure 1 for LC3B survival curve).

According to work published by Iwade et al. and Liu et al. we subclassified our cohort based on p62 and LC3B expression and subcellular localization of p62 as described in the Materials and Methods section $[12,13]$. The p62 cytoplasmic/p62 nuclear LL category correlated with the worst overall survival, the p62 cytoplasmic/p62 nuclear HH category with the best overall survival, while both the HL and LH categories showed intermediate survival rates ( $p=0.003$, data not shown), which justified a combination of these groups into a "mixed" group (Figure 6A). Similarly, the LC3B/p62 LL group faired the worse when compared to $\mathrm{HH}$ and mixed subtypes $(p=$ 0.013 , Figure 6B). When comparing the LL group with the remainder of all other cases grouped together it also showed the worse overall survival ( $p=0.005$, data not shown).

Other prognostic relevant parameters were $\mathrm{pT}$ category $(p=0.001), \mathrm{pN}$ category $(p=0.002)$, presence of distant metastases $(p=0.045)$, lymphatic vessel invasion ( $p=0.034)$, and UICC staging $(p=0.001)$, and in trend the presence of perineural invasion $(p=0.058)$, but not resection status, venous invasion and intratumoral inflammation $(p=0.485, p=0.434$ and $p=0.406$ respectively). 
Table 4: p62 dot-like, p62 cytoplasmic and p62 nuclear staining patterns correlated to clinicopathological features respectively.

\begin{tabular}{|c|c|c|c|c|c|c|c|c|c|c|c|}
\hline \multirow{2}{*}{ Parameter } & \multirow{2}{*}{ Category } & \multirow{2}{*}{ Total } & \multicolumn{3}{|c|}{ p62 dots } & \multicolumn{3}{|c|}{ p62 cytoplasm } & \multicolumn{3}{|c|}{ p62 nuclear } \\
\hline & & & Low & High & $p$-value & Low & High & $p$-value & Low & High & $p$-value \\
\hline pT category & $\begin{array}{l}\text { pT1 } \\
\text { pT2 } \\
\text { pT3 } \\
\text { pT4 }\end{array}$ & \begin{tabular}{|l|}
35 \\
11 \\
67 \\
3 \\
\end{tabular} & $\begin{array}{l}11 \\
6 \\
30 \\
1 \\
\end{array}$ & \begin{tabular}{|l}
24 \\
5 \\
37 \\
2 \\
\end{tabular} & 0.455 & \begin{tabular}{|l|}
0 \\
1 \\
11 \\
0
\end{tabular} & \begin{tabular}{|l}
35 \\
10 \\
56 \\
3
\end{tabular} & 0.070 & \begin{tabular}{|l|}
12 \\
6 \\
38 \\
2 \\
\end{tabular} & \begin{tabular}{|l|}
23 \\
5 \\
29 \\
1
\end{tabular} & 0.165 \\
\hline $\begin{array}{l}\text { Lymph Node } \\
\text { Metastasis }\end{array}$ & $\begin{array}{l}\text { absent } \\
\text { present }\end{array}$ & $\begin{array}{l}56 \\
60 \\
\end{array}$ & $\begin{array}{l}23 \\
25\end{array}$ & $\begin{array}{l}33 \\
35\end{array}$ & 1.000 & \begin{tabular}{|l}
5 \\
7 \\
\end{tabular} & $\begin{array}{l}51 \\
53\end{array}$ & 0.764 & $\begin{array}{l}21 \\
37\end{array}$ & $\begin{array}{l}35 \\
23\end{array}$ & 0.015 \\
\hline $\begin{array}{l}\text { Distant } \\
\text { Metastasis }\end{array}$ & $\begin{array}{l}\text { absent } \\
\text { present }\end{array}$ & $\begin{array}{l}111 \\
5\end{array}$ & $\begin{array}{l}46 \\
2\end{array}$ & $\begin{array}{l}65 \\
3\end{array}$ & 1.000 & \begin{tabular}{|l|}
12 \\
0
\end{tabular} & $\begin{array}{l}99 \\
5\end{array}$ & 1.000 & $\begin{array}{l}54 \\
4\end{array}$ & $\begin{array}{l}57 \\
1\end{array}$ & 0.364 \\
\hline UICC Stage & \begin{tabular}{|l} 
I \\
II \\
III \\
IV
\end{tabular} & \begin{tabular}{|l|}
35 \\
25 \\
51 \\
5 \\
\end{tabular} & $\begin{array}{l}14 \\
11 \\
21 \\
2\end{array}$ & \begin{tabular}{|l|}
21 \\
14 \\
30 \\
3 \\
\end{tabular} & 0.991 & $\begin{array}{l}0 \\
6 \\
6 \\
0\end{array}$ & \begin{tabular}{|l|}
35 \\
19 \\
45 \\
5 \\
\end{tabular} & 0.021 & \begin{tabular}{|l|}
11 \\
13 \\
30 \\
4
\end{tabular} & \begin{tabular}{|l|}
24 \\
12 \\
21 \\
1
\end{tabular} & 0.041 \\
\hline $\begin{array}{l}\text { Lymphatic } \\
\text { Invasion }\end{array}$ & $\begin{array}{l}\text { absent } \\
\text { present }\end{array}$ & $\begin{array}{l}38 \\
78\end{array}$ & $\begin{array}{l}18 \\
30\end{array}$ & $\begin{array}{l}20 \\
48\end{array}$ & 0.423 & \begin{tabular}{|l}
4 \\
8
\end{tabular} & $\begin{array}{l}34 \\
70\end{array}$ & 1.000 & $\begin{array}{l}18 \\
40\end{array}$ & $\begin{array}{l}20 \\
38\end{array}$ & 0.843 \\
\hline Venous Invasion & $\begin{array}{l}\text { absent } \\
\text { present }\end{array}$ & $\begin{array}{l}87 \\
29 \\
\end{array}$ & $\begin{array}{l}39 \\
9\end{array}$ & $\begin{array}{l}48 \\
20\end{array}$ & 0.276 & $\begin{array}{l}8 \\
4\end{array}$ & $\begin{array}{l}79 \\
25 \\
\end{array}$ & 0.491 & $\begin{array}{l}41 \\
17 \\
\end{array}$ & $\begin{array}{l}46 \\
12\end{array}$ & 0.391 \\
\hline $\begin{array}{l}\text { Perineural } \\
\text { Invasion }\end{array}$ & $\begin{array}{l}\text { absent } \\
\text { present }\end{array}$ & $\begin{array}{l}70 \\
45\end{array}$ & $\begin{array}{l}29 \\
19\end{array}$ & $\begin{array}{l}42 \\
26\end{array}$ & 1.000 & \begin{tabular}{|l}
7 \\
5
\end{tabular} & $\begin{array}{l}64 \\
40\end{array}$ & 1.000 & $\begin{array}{l}31 \\
27\end{array}$ & $\begin{array}{l}40 \\
18\end{array}$ & 0.127 \\
\hline Inflammation & $\begin{array}{l}\text { low } \\
\text { high }\end{array}$ & $\begin{array}{l}55 \\
61\end{array}$ & $\begin{array}{l}26 \\
22\end{array}$ & $\begin{array}{l}29 \\
39\end{array}$ & 0.260 & $\begin{array}{l}4 \\
8 \\
\end{array}$ & $\begin{array}{l}51 \\
53 \\
\end{array}$ & 0.370 & $\begin{array}{l}26 \\
32 \\
\end{array}$ & $\begin{array}{l}29 \\
29\end{array}$ & 0.710 \\
\hline Grading & $\begin{array}{l}\text { G1 } \\
\text { G2 } \\
\text { G3 }\end{array}$ & \begin{tabular}{|l|}
18 \\
50 \\
48
\end{tabular} & $\begin{array}{l}8 \\
22 \\
18\end{array}$ & \begin{tabular}{|l}
10 \\
28 \\
30
\end{tabular} & 0.886 & \begin{tabular}{|l|}
0 \\
5 \\
7
\end{tabular} & $\begin{array}{l}18 \\
45 \\
41\end{array}$ & 0.322 & \begin{tabular}{|l|}
9 \\
18 \\
31
\end{tabular} & $\begin{array}{l}9 \\
32 \\
17\end{array}$ & 0.042 \\
\hline Resection Status & $\begin{array}{l}\text { R0 } \\
\text { R1 }\end{array}$ & $\begin{array}{l}109 \\
7\end{array}$ & $\begin{array}{l}43 \\
5\end{array}$ & $\begin{array}{l}66 \\
2\end{array}$ & 0.124 & \begin{tabular}{|l|}
11 \\
1
\end{tabular} & $\begin{array}{l}98 \\
6\end{array}$ & 0.544 & $\begin{array}{l}56 \\
2\end{array}$ & $\begin{array}{l}53 \\
5\end{array}$ & 0.438 \\
\hline
\end{tabular}

Statistically significant $p$ values are shown in bold.

In a multivariate analysis encompassing the relevant prognostic factors $\mathrm{pT}$ category, lymph node and distant metastases, grading, lymphatic and perineural invasion, the classification strategy of p62 cytoplasmic/p62 nuclear (where cytoplasmic also includes dot-like staining) showed the highest significance level for prognostic discrimination and was an independent prognostic parameter $(p=0.033$, HR $=0.561 ;$ Table 6). p62 cytoplasmic/p62 nuclear staining was also independent prognostic factor in a model encompassing tumor grading and UICC stage $(p=0.021$; Table 7).

\section{DISCUSSION}

In the present study we aimed to assess the role of basal autophagy in EAC. We observed intact basal autophagy in EAC in vitro as we observed a dosedependent increase of markers LC3B-II and p62 with increasing concentrations of autophagy inhibitor CQ. CQ prevents the fusion of autophagosomes and lysosomes and subsequent degradation. As LC3B-II and p62 is incorporated into the autophagosomal membranes and subsequently degraded, inhibition at a late stage will result in accumulation of these markers and is indicative of functional basal autophagy.

Low p62 staining patterns, regardless of type, subcellular localization or combination with LC3B, correlated with a worse prognosis. Of note, low p62 nuclear staining was associated with lymph node metastases, a higher grade and with higher stage tumors. There was no intratumoral heterogeneity of the staining patterns of our autophagy related markers and no correlation between them and intratumoral inflammatory infiltrates. This was unexpected following the concept that autophagy may be strongly linked to inflammation and may show specific reaction patterns to different environment such as the tumor periphery in contrast to the tumor center $[14,15]$. Regarding survival, the classification of our cohort based on p62 cytoplasmic/p62 nuclear staining patterns was an independent prognostic factor with a significantly worse outcome in patients with low cytoplasmic/low nuclear $\mathrm{p} 62$ expressing tumors.

At present the literature focusing on the role of autophagy in EAC is limited. O'Donovan et al. [16] reported that chemoresistant esophageal cancer cell lines induce cytoprotective autophagy upon treatment. A recent follow up study [17] added that pharmacological autophagy inducers rapamycin and lithium had diverging effects when combined with chemotherapeutic agents in esophageal cancer cell lines. Given the different research settings it is difficult to situate our current work in relation to the aforementioned studies. Roesley et al. [18] 
Table 5: p62 sum score and clinicopathological features.

\begin{tabular}{|c|c|c|c|c|c|}
\hline Parameter & Category & Total & p62 Sum Low & p62 Sum High & $p$-value \\
\hline pT category & \begin{tabular}{|l|} 
pT1 \\
pT2 \\
pT3 \\
pT4
\end{tabular} & \begin{tabular}{|l|}
35 \\
11 \\
67 \\
3
\end{tabular} & \begin{tabular}{|l|}
2 \\
2 \\
18 \\
0
\end{tabular} & $\begin{array}{l}33 \\
9 \\
49 \\
3\end{array}$ & 0.059 \\
\hline $\begin{array}{ll}\text { Lymph } & \text { Node } \\
\text { Metastasis } & \\
\end{array}$ & $\begin{array}{l}\text { absent } \\
\text { present }\end{array}$ & $\begin{array}{l}56 \\
60\end{array}$ & $\begin{array}{l}9 \\
13 \\
\end{array}$ & $\begin{array}{l}47 \\
47\end{array}$ & 0.485 \\
\hline Distant Metastasis & $\begin{array}{l}\text { absent } \\
\text { present }\end{array}$ & $\begin{array}{l}111 \\
5\end{array}$ & $\begin{array}{l}20 \\
2\end{array}$ & $\begin{array}{l}91 \\
3 \\
\end{array}$ & 0.239 \\
\hline UICC Stage & \begin{tabular}{|l} 
I \\
II \\
III \\
IV \\
\end{tabular} & \begin{tabular}{|l|}
35 \\
25 \\
51 \\
5 \\
\end{tabular} & $\begin{array}{l}2 \\
9 \\
9 \\
2 \\
\end{array}$ & \begin{tabular}{|l|}
33 \\
16 \\
42 \\
3 \\
\end{tabular} & 0.017 \\
\hline $\begin{array}{l}\text { Lymphatic } \\
\text { Invasion }\end{array}$ & $\begin{array}{l}\text { absent } \\
\text { present }\end{array}$ & $\begin{array}{l}38 \\
78\end{array}$ & $\begin{array}{l}7 \\
15 \\
\end{array}$ & $\begin{array}{l}31 \\
63\end{array}$ & 1.000 \\
\hline Venous Invasion & $\begin{array}{l}\text { absent } \\
\text { present }\end{array}$ & $\begin{array}{l}87 \\
29\end{array}$ & $\begin{array}{l}15 \\
7\end{array}$ & $\begin{array}{l}72 \\
22\end{array}$ & 0.421 \\
\hline Perineural Invasion & $\begin{array}{l}\text { absent } \\
\text { present }\end{array}$ & $\begin{array}{l}71 \\
45 \\
\end{array}$ & $\begin{array}{l}11 \\
11\end{array}$ & $\begin{array}{l}60 \\
34\end{array}$ & 0.237 \\
\hline Inflammation & $\begin{array}{l}\text { low } \\
\text { high }\end{array}$ & $\begin{array}{l}55 \\
61 \\
\end{array}$ & \begin{tabular}{|l|}
14 \\
8 \\
\end{tabular} & $\begin{array}{l}41 \\
53 \\
\end{array}$ & 0.102 \\
\hline Grading & $\begin{array}{l}\mathrm{G} 1 \\
\mathrm{G} 2 \\
\mathrm{G} 3\end{array}$ & $\begin{array}{l}18 \\
50 \\
48 \\
\end{array}$ & \begin{tabular}{|l|}
3 \\
7 \\
12 \\
\end{tabular} & $\begin{array}{l}15 \\
43 \\
36\end{array}$ & 0.415 \\
\hline Resection Status & $\begin{array}{l}\text { R0 } \\
\text { R1 }\end{array}$ & \begin{tabular}{|l|}
109 \\
7
\end{tabular} & $\begin{array}{l}20 \\
2\end{array}$ & $\begin{array}{l}89 \\
5\end{array}$ & 0.616 \\
\hline
\end{tabular}

Statistically significant $p$ values are shown in bold.

Table 6: p62 cytoplasmic/p62 nuclear classification was shown to be an independent prognostic parameter in a multivariate analysis encompassing $\mathrm{pT}$ category, $\mathrm{pN}$ category, absence or presence of distant metastasis, lymphatic invasion and perineural invasion as well as grading.

\begin{tabular}{|l|l|l|l|l|}
\hline \multirow{2}{*}{ parameter } & \multirow{2}{*}{ HR } & \multicolumn{2}{|l|}{$\mathbf{9 5 \%}$ confidence interval } & \multirow{2}{*}{ p-value } \\
\cline { 3 - 4 } & & $\mathbf{m i n}$ & $\mathbf{m a x}$ & \\
\hline $\begin{array}{l}\text { pT category } \\
\text { pT1 vs pT2 vs pT3 vs pT4 }\end{array}$ & 1.505 & 0.916 & 2.472 & 0.106 \\
\hline $\begin{array}{l}\text { pN category } \\
\text { pN0 vs pN1 vs pN2 vs pN3 }\end{array}$ & 1.396 & 0.985 & 1.977 & 0.061 \\
\hline $\begin{array}{l}\text { distant metastases } \\
\text { absent } v s \text { present }\end{array}$ & 1.684 & 0.464 & 6.110 & 0.428 \\
\hline $\begin{array}{l}\text { lymphatic invasion } \\
\text { absent } v s \text { present }\end{array}$ & 1.021 & 0.374 & 2.783 & 0.968 \\
\hline $\begin{array}{l}\text { perineural invasion } \\
\text { absent } v \text { present }\end{array}$ & 0.502 & 0.211 & 1.195 & 0.119 \\
\hline $\begin{array}{l}\text { Grading } \\
\text { G1 } v s \text { G2 } v s \text { G3 }\end{array}$ & 1.584 & 0.935 & 2.685 & 0.087 \\
\hline $\begin{array}{l}\text { p62 cyto/p62 nucl } \\
\text { LL } v s \text { mixed/HH }\end{array}$ & 0.561 & 0.329 & 0.956 & $\mathbf{0 . 0 3 3}$ \\
\hline
\end{tabular}

Statistically significant $p$-values are shown in bold. HR - Hazard Ratio

reported that dysplastic $\mathrm{BE}$ and $\mathrm{EAC}$ have lower levels of Beclin-1, an early autophagy initiator, when compared to non-dysplastic BE and normal squamous mucosa. This contrasts with our study as we observed significantly lower levels of LC3B and p62 in normal esophagus and gastric mucosa, when compared to the tumor tissue in our cohort. We found the autophagy machinery in EAC under basal conditions to be intact in vitro. Preliminary experiments performed in our group confirmed that pharmacological and amino acid starvation autophagy induction in EAC cell lines OE19 and OE33 (data not shown). This is in contrast to previous reports by Roesley et al., stating that dysplastic BE and EAC displays resistance to autophagy inducers. These discrepancies can be explained by 
Table 7: p62 cytoplasmic/p62 nuclear classification was shown to be an independent prognostic parameter in a multivariate analysis encompassing UICC and grading.

\begin{tabular}{|l|l|l|l|l|}
\hline \multirow{2}{*}{ Parameter } & \multirow{2}{*}{ HR } & 95\% confidence interval & \multirow{2}{*}{-value } \\
& & min & max & \\
\hline $\begin{array}{l}\text { UICC stage } \\
\text { I } v \text { II } v \text { III } v s \text { IV }\end{array}$ & 1.792 & 1.198 & 2.682 & $\mathbf{0 . 0 0 5}$ \\
\hline $\begin{array}{l}\text { Grading } \\
\text { G1 } v s \text { G2 } v s \text { G3 }\end{array}$ & 1.451 & 0.885 & 2.380 & 0.140 \\
\hline $\begin{array}{l}\text { p62 cyto/p62 nucl } \\
\text { LL } v s \text { mixed/HH }\end{array}$ & 0.549 & 0.330 & 0.914 & $\mathbf{0 . 0 2 1}$ \\
\hline
\end{tabular}

Statistically significant $p$-values are shown in bold. HR - Hazard Ratio

A

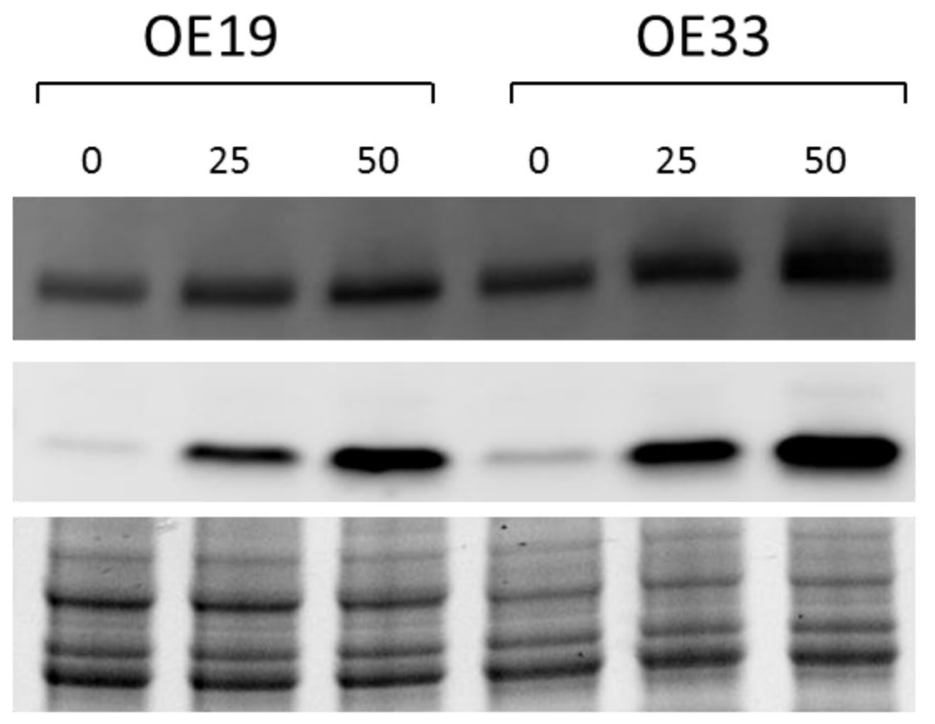

$\mathrm{CQ}(\mu \mathrm{M})$

p62 (62kDa)

LC3B-I (16kDa)

LC3B-II (14kDa)

Total Protein

B
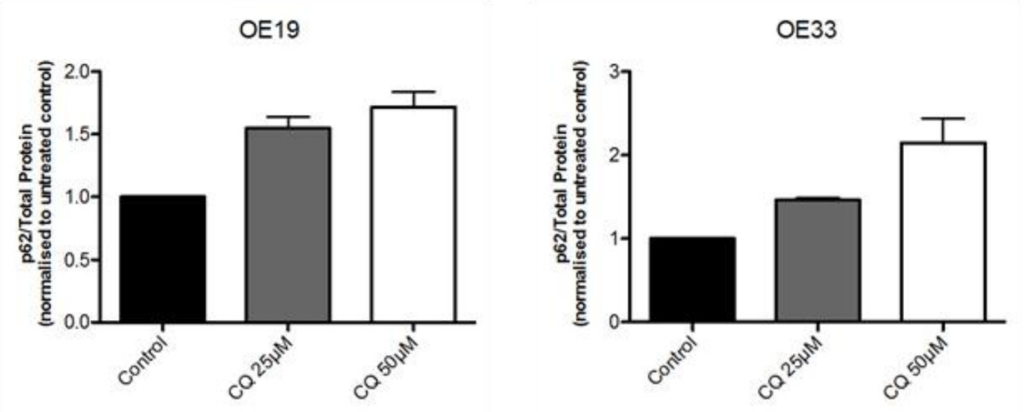

OE19
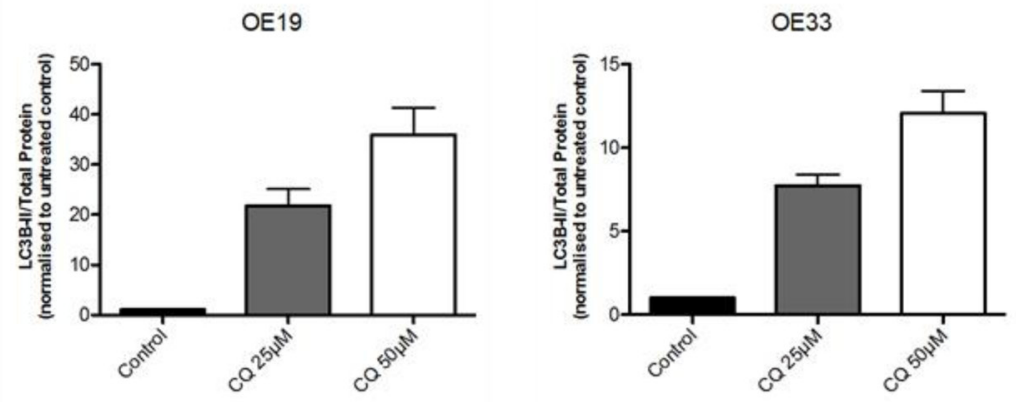

Figure 1: EAC exhibits intact basal autophagy in vitro as determined by immunoblotting of markers LC3B and p62 upon pharmacological autophagy inhibition. A. EAC cell lines OE19 and OE33 were treated with increasing concentrations of the autophagy inhibitor chloroquine (CQ) for $48 \mathrm{hr}$ followed by immunoblotting of the autophagy markers p62 and LC3B. Total protein was visualized as loading control. Representative blot is shown. B. Quantification of triplicate repetitions of experiments shown in A. 
different experimental set ups being used in previous work that may not adequately discriminate between an induction or a block of autophagy. We observed that lower levels of LC3B and p62 correlated with more advanced staged tumors. In addition we observed higher levels of both markers, when compared to normal matched tissue, in a small sample collection of non-dysplastic and dysplastic BE tissue (data not shown). Although we did not explicitly investigate the role of autophagy in the progression of Barrett's esophagus, these preliminary findings are indicative for an important role of autophagy in EAC pathogenesis and warrant further investigation.
El-Mashed et al. identified LC3B cytoplasmic, LC3B ring-like structures and LC3B stone-like structures in treatment naïve and neoadjuvant treated EAC cohorts [19]. LC3B cytoplasmic staining correlated with a better overall survival, which is contrast to our study as low LC3B dot-like structures, in combination with low p62 expression, correlated with a shorter overall survival. We did not observe globular structures, while only observing ring-like structures in a very small subset of cases without prognostic significance. The difference in staining patterns observed could be explained by the different specificity and reactivity of the antibodies used in each study. El-

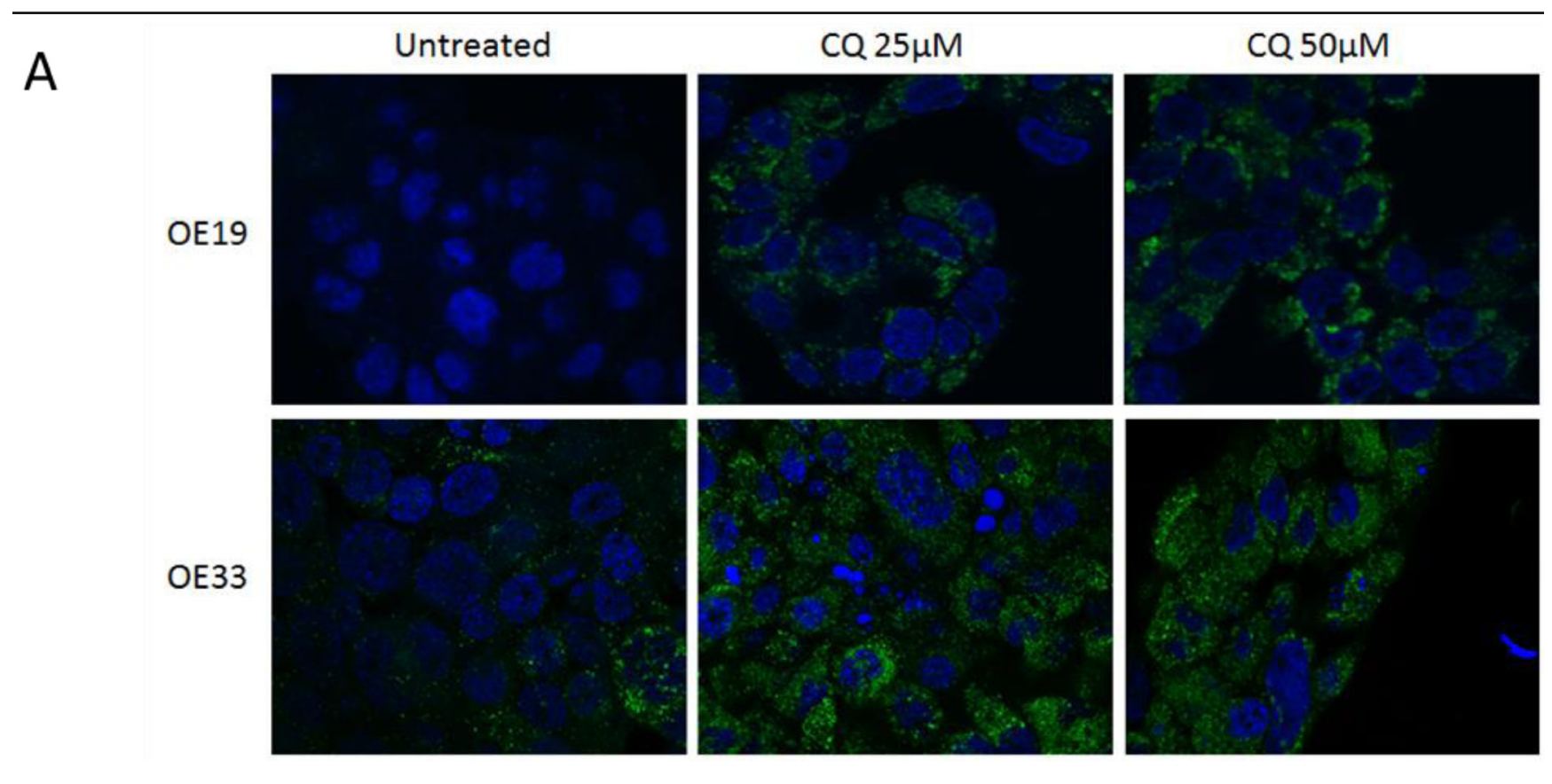

B

OE19

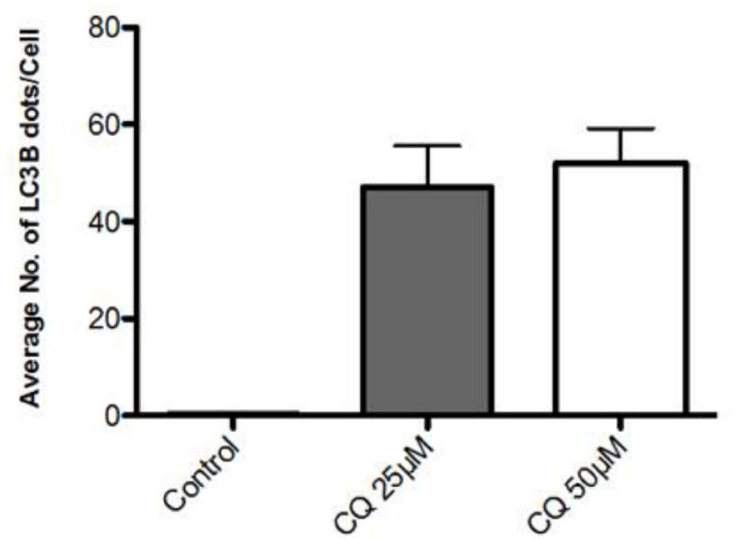

OE33

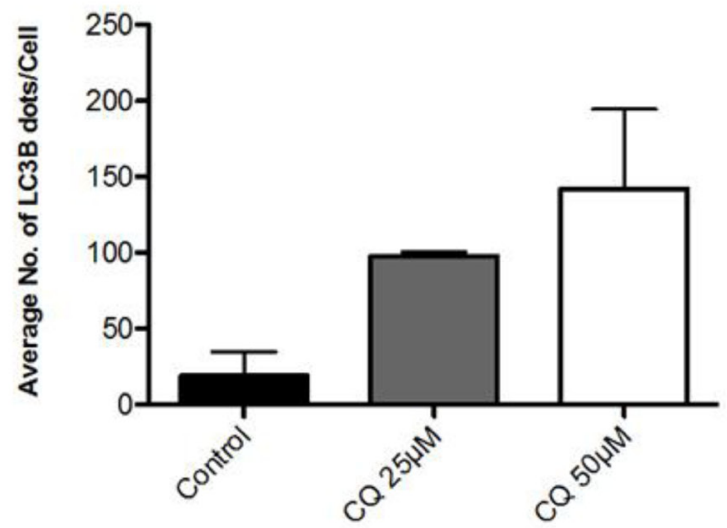

Figure 2: EAC exhibits intact basal autophagy in vitro as determined by immunofluorescent staining of LC3B upon pharmacological autophagy inhibition. A. OE19 and OE33 were treated with increasing concentrations of CQ for 48hr and LC3B detected via immunofluorescence. LC3B-II is visualized as green punctae, representing autophagosomes. Nuclei were stained with DAPI (blue). Representative images are shown. B. Quantification of triplicate repetitions of experiments shown in A. 


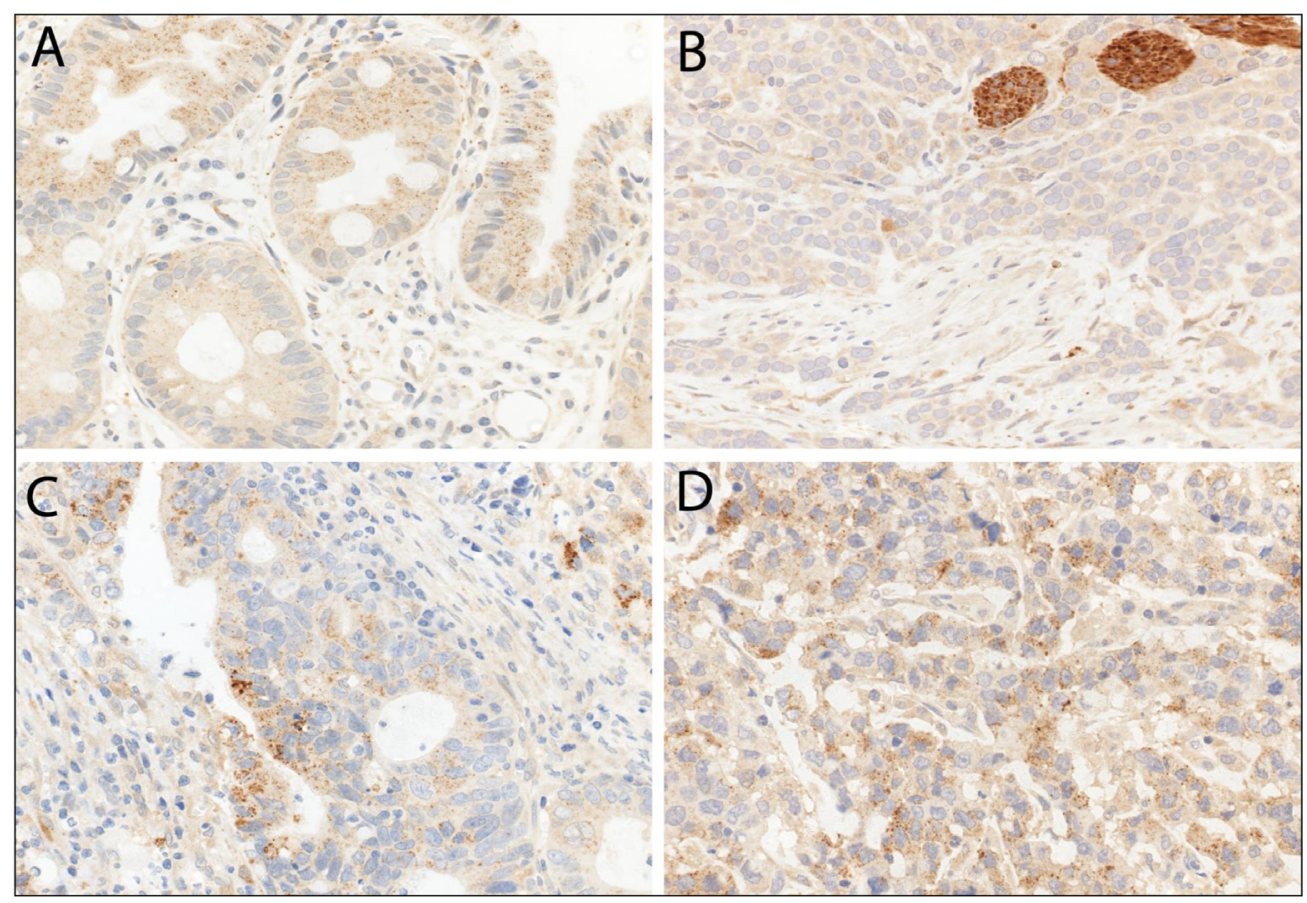

Figure 3: LC3B immunohistochemical staining of Barrett's esophagus (BE) and esophageal adenocarcinoma (EAC). A. BE tissue with goblet cells and high LC3B dot-like staining. B. EAC tissue with low LC3B dot-like staining and nerves as internal positive control. C. and D. EAC tissue with high LC3B dot-like staining. Representative images were taken on a Zeiss Axioskop microscope at 40X objective magnification and corrected for brightness.

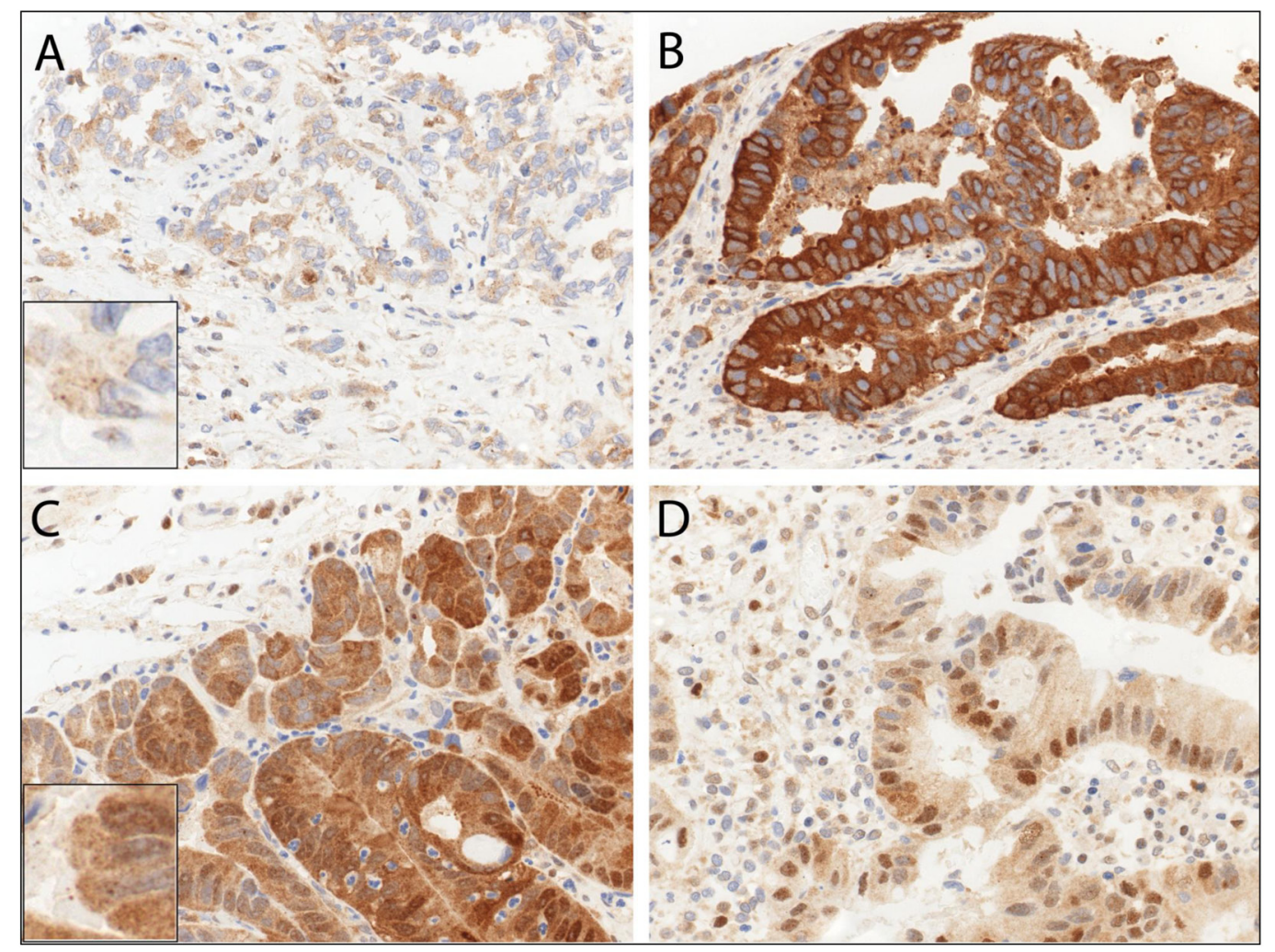

Figure 4: Immunohistochemical staining patterns of p62 in esophageal adenocarcinoma (EAC). EAC tissue exhibiting A. low p62 cytoplasmic staining, B. high p62 cytoplasmic staining C. both high p62 dot-like and diffuse cytoplasmic staining, and D. high p62 nuclear positivity. Representative images were taken on a Zeiss Axioskop microscope at 40X objective magnification and corrected for brightness. Insert in $\mathbf{A}$. and $\mathbf{C}$. is for better visualization of the hardly visible dots. 
Mashed et al. used anti-LC3B antibody from Abgent (rabbit polyclonal, directed at N-terminal 1-30 amino acids, AP1802a), while anti-LC3B antibody from Novus Biologicals (rabbit polyclonal, directed at N-terminal \#NB600-1384) was used in our study. It has also been published that the anti-LC3B antibody from Novus Biologicals shows crossreactivity with LC3A [20]. It has been demonstrated that the different isoforms of LC3 is differentially expressed in various tissues, however little is known about the expression patterns in esophageal normal and neoplastic tissue. Furthermore, it has been shown that both LC3A and LC3B localized to autophagosomal membranes upon induction and are therefore suitable markers [21, 22]. While the crossreactivity of the antibody in this study can be considered a confounding factor, the

A

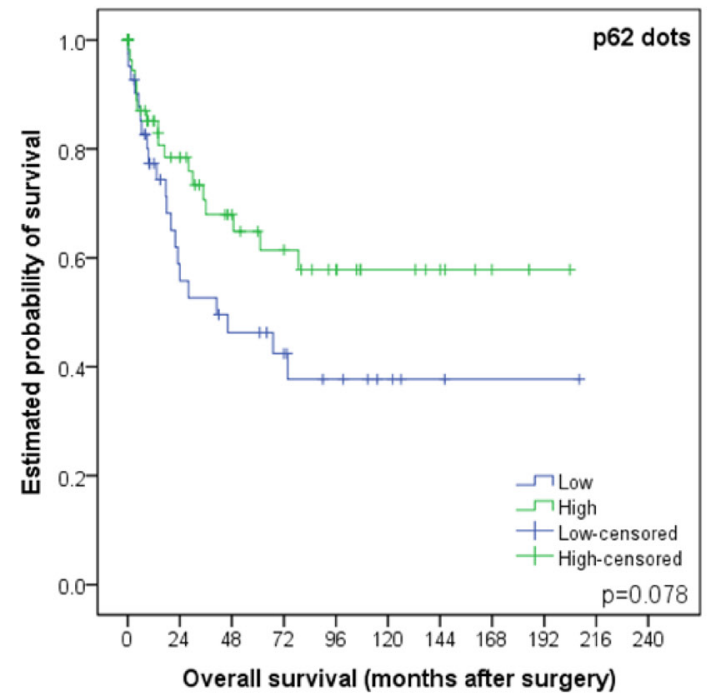

C

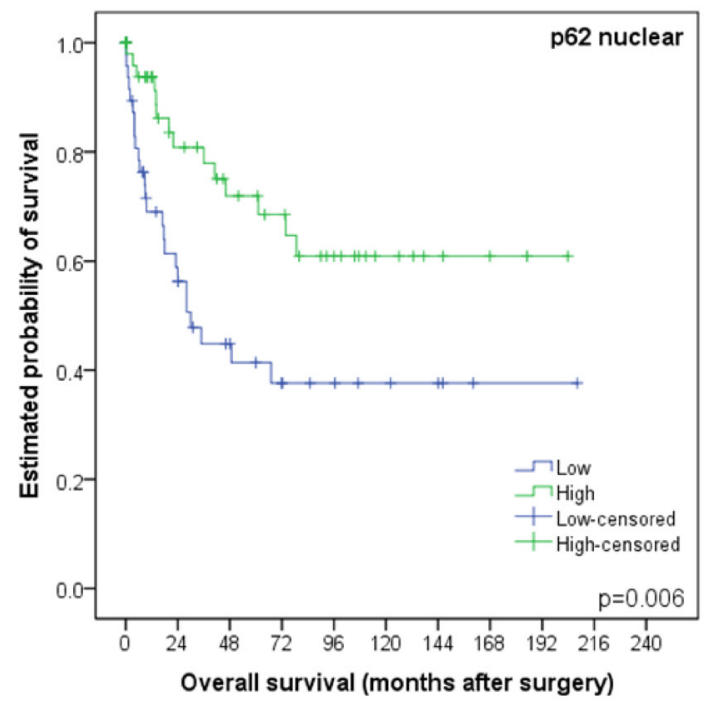

interpretation of staining patterns remains the same as both isoforms are markers for autophagic activity. This highlights the importance of standardization of staining and scoring protocols of LC3B prior to widespread implementation as a routine biomarker.

Our finding that low p62 expression correlates with a more aggressive phenotype, worse prognosis and worse overall survival in EAC is in contrast to numerous studies done on other tumor entities [23-27], although there are also diverging reports about a potential prognostic impact of p62 [13]. At present, p62's role in cancer has to be considered context dependent and elucidating the underlying mechanism is complicated given the multiple functions of $\mathrm{p} 62$.

B

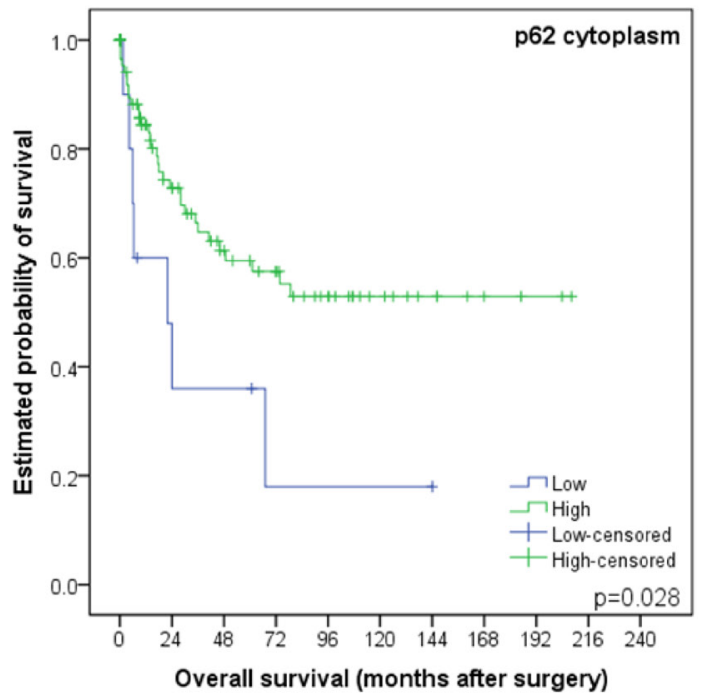

D

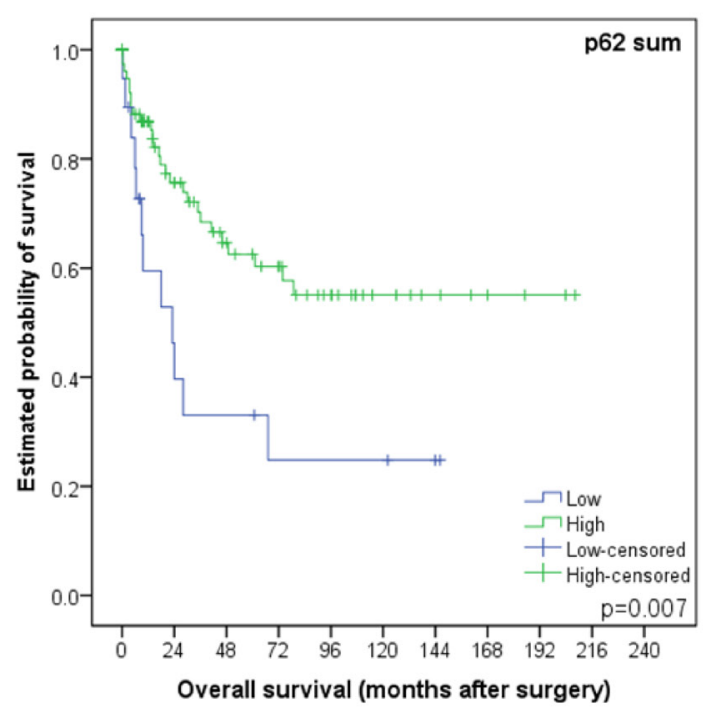

Figure 5: Kaplan-Meier survival curves for p62 staining patterns in esophageal adenocarcinomas. A. Dot-like, B. cytoplasmic or C. nuclear staining classified as either low or high. D. p62 sum score. For each curve the p-value is displayed on the bottom right-hand corner. 
It is well described that p62 functions as an adaptor protein, which binds ubiquitinated protein aggregates and delivers it to autophagosomes via its association with LC3B [28]. In addition, p62 has been identified as a major player in other pathways. This includes the nuclear factor erytheriod-derived-2-like 2 -Kelch-like ECH-associated protein 1-antioxidant response element (Nrf2-Keap1ARE) pathway, where dysregulated p62 may contribute to oncogenesis $[9,23]$. p62 also plays a tumor promoting role via the nuclear factor kappa $\mathrm{B}(\mathrm{NF}-\kappa \mathrm{B})$ pathway and increased inflammation [29-31]. Given that low cytoplasmic p62 correlates with a worse overall survival in our EAC cohort it seems unlikely that these pathways are contributing factors. p62 has also been described as a tumor suppressor as it facilitates autophagic degradation of regulators of the oncogenic Wnt signalling pathway [32]. It is therefore tempting to speculate that this could be an underlying mechanism for the prognostic impact observed in our cohort. Furthermore, p62 has been shown to be a regulatory player in mitosis and cell cycle regulation [3335], and has also been described to mediate an alternative nuclear proteolytic degradation pathway [36]. One may speculate that the cases with low nuclear p62 expression, that were associated with a worse prognosis suffered from more nuclear instability/increased nuclear protein aggregation, which contributed to disease progression and an aggressive phenotype.

To our knowledge this is the first study to investigate the prognostic relevance of both autophagy markers $\mathrm{LC} 3 \mathrm{~B}$ and p62 in primary resected EAC. Investigating

A

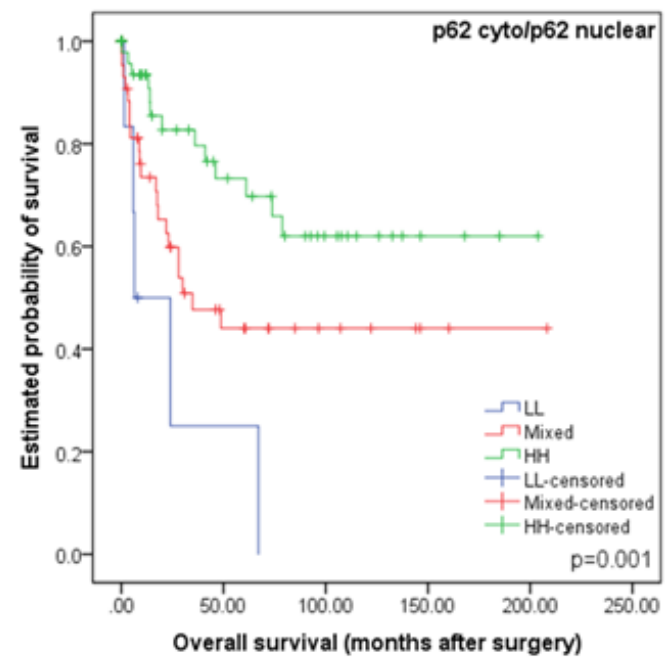

the underlying mechanism as to why low p62 expression consistently correlated with a worse patient outcome could lead to a better understanding of EAC biology and open up novel avenues for therapeutic intervention. Basal autophagy, as indicated by low LC3B and p62 expression, may give tumors access to a continuous supply of nutrients and building blocks thereby facilitating progression and an aggressive phenotype. Late stage impairment of basal or activated autophagy, mirrored by mixed or high LC3B and p62 expression, would not confer this oncogenic advantage. Given the divergent roles of autophagy described in neoplastic diseases this is a feasible hypothesis to explain our results. We are aware that assessing the dynamic process of autophagy using a static IHC ex vivo approach is a limitation. However, taken together the results described in this study highlight the potential role of autophagy, and other p62 related pathways, in EAC biology and warrant further investigation.

\section{MATERIALS AND METHODS}

\section{Cell lines, culture and treatment conditions}

The human EAC cancer cell lines OE19 and OE33 from the Public Health England Culture Collections were obtained via Sigma-Aldrich, Buchs, Switzerland. The cell lines were cultured in RPMI-1640 (Sigma-Aldrich, Buchs, Switzerland) supplemented with $10 \%$ fetal bovine serum

\section{B}

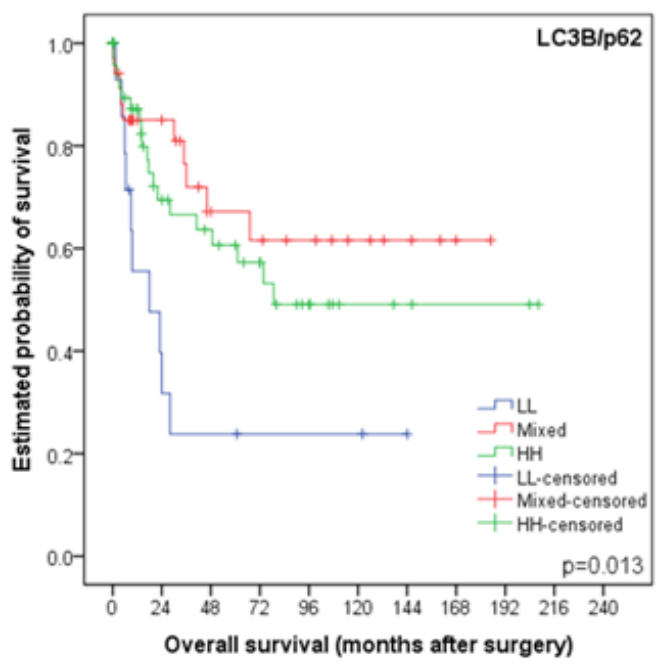

Figure 6: Kaplan-Meier survival curves for subcellular location patterns of autophagy markers p62 and LC3B in esophageal adenocarcinomas. A. Kaplan-Meier survival curve analysis using classification strategy based on subcellular location and expression of p62. The cohort was subdivided into three groups based on their p62 cytoplasmic (including dot-like) and nuclear staining patterns: low p62 cytoplasmic/low p62 nuclear (LL), either low p62 cytoplasmic/high p62 nuclear or vice versa (mixed), high p62 cytoplasmic/high p62 nuclear (HH). B. Kaplan-Meier survival curve analysis using classification strategy based on LC3B dot-like expression and total p62 expression. The cohort was subdivided into three groups: low LC3B/low p62 (LL), either low LC3B/high p62 or vice versa (mixed), high LC3B/high p62 (HH). For each curve the p-value is displayed on the bottom right-hand corner. 
(Sigma-Aldrich, F7524) and 1\% penicillin streptomycin. Chloroquine (Sigma-Aldrich, C6628) was dissolved in distilled water and stored at $-20^{\circ} \mathrm{C}$. Chloroquine (CQ) inhibits late stage autophagy by increasing lysosomal $\mathrm{pH}$ thereby preventing the autophagosome-lysosome fusion and subsequent degradation of autophagic flux markers LC3B-II and p62. Lipidated form of LC3B-II migrates faster than the unlipidated LC3B-I on an SDS-PAGE gel and therefore the two isoforms can be separated and visualized using immunoblotting. Cells were seeded in a 6 well dish and allowed to adhere overnight and CQ was added to the experimental wells the following day at final concentrations of $25 \mu \mathrm{M}$ and $50 \mu \mathrm{M}$ and incubated for $48 \mathrm{hr}$ prior to harvesting or fixing of cells for subsequent immunoblotting or immunofluorescence respectively.

\section{Immunoblotting}

OE19 and OE33 cells were washed in phosphate buffered saline (PBS) before lysis in urea buffer ( $8 \mathrm{M}$ urea, $0.5 \%$ tritonX) containing protease inhibitor (complete midi, Roche Diagnostics, Rotkreuz, Switzerland). Samples were then sonicated and centrifuged at 13,000 rcf for 30min. Supernatant was subsequently transferred to a fresh tube. Protein concentration was determined using the Bradford protein assay (BioRad, Cressier, Switzerland). Thirty $\mu$ g of total protein were denatured in selfmade $5 \mathrm{X}$ sample buffer with $\beta$-mercaptoethanol (Sigma Aldrich, M-7522) at $95^{\circ} \mathrm{C}$ for $5 \mathrm{~min}$. Samples were loaded on a $4-20 \%$ stain-free pre-cast gel (BioRad) and transferred onto a polyvinylidene difluoride membrane using the Trans-Blot ${ }^{\circledR}$ Turbo $^{\text {TM }}$ Transfer system (BioRad). Total protein was visualized as loading control using the ChemiDoc $^{\text {TM }}$ MP system (BioRad). Primary antibodies were purchased as follows: the anti-LC3B antibody from Novus Biologicals (rabbit polyclonal, \#NB600-1384, LuBioScience, Luzern, Switzerland) and the anti-p62/ SQSTM1 antibody from Sigma (mouse monoclonal, clone 2C11, \#WH0008878M1, Leiden, The Netherlands). For p62 membranes were blocked in 5\% milk/tris-buffered saline (TBS) for $1 \mathrm{hr}$ at room temperature (RT), while membranes for LC3B were blocked in 5\% bovine serum albumin (BSA)/TBS for $1 \mathrm{hr}$ at RT. Working solutions of the primary LC3B and p62 antibodies were prepared with final dilution $1: 1000$ in 5\% milk/TBS with $0.1 \%$ Tween (Sigma Aldrich, P9416). Membranes were incubated overnight at $4^{\circ} \mathrm{C}$ with shaking. DyLight ${ }^{\circledR} 650$ conjugated goat anti-rabbit or DyLight ${ }^{\circledR} 550$ conjugated goat antimouse (LabForce, Muttenz, Switzerland) was used as secondary antibodies and diluted 1:1000 in 5\% milk/ TBS-T. Membranes were incubated for $1 \mathrm{hr}$ at RT with shaking. Proteins of interest were acquired and visualized using the ChemiDoc ${ }^{\mathrm{TM}}$ MP system (BioRad). Bands were quantified using ImageJ software (1.64r; NIH, Bethesda, MD, USA).

\section{Immunofluorescence}

OE19 and OE33 was seeded directly onto coverslides $(18 \mathrm{mmX} 18 \mathrm{~mm})$ in a 6 well plate and allowed to adhere overnight. Cells remained untreated or treated with $25 \mu \mathrm{M}$ and $50 \mu \mathrm{M}$ Chloroquine (CQ) for $48 \mathrm{hr}$ prior to fixation. Cells were washed in PBS, pre-fixed in $4 \%$ paraformaldehyde for 15 seconds, fixed and permeabilized in ice-cold $100 \%$ methanol for $10 \mathrm{~min}$ at $-20^{\circ} \mathrm{C}$. Slides were air dried and washed with PBS prior to incubation with the primary anti-LC3B antibody (Cell Signaling, Lausen, Switzerland, rabbit monoclonal,clone D11, \#3686,) at a working dilution of 1:100 in PBS/1\%BSA/0.1\%Tween for $1 \mathrm{hr}$ at RT. Subsequently slides were washed twice with PBS $0.1 \%$ Tween and once with PBS and incubated for $1 \mathrm{hr}$ at RT with the fluorescein isothiocyanate (FITC) conjugated antirabbit secondary antibody (\#111-096-045 Jackson Immunoresearch, Suffolk, UK) in a working dilution of $1: 130$ in PBS/1\%BSA/0.1\% Tween. Slides were once again washed twice with PBS $0.1 \%$ Tween and once with PBS. Slides were allowed to air dry. Mounting media (Thermofisher Scientific, Reinach, Switzerland, S36938) containing DAPI (4',6-diamidino2-phenylindole) was added to slides in order to visualize nuclei. Images were taken on a confocal microscope Olympus FluoView1000 at 63x objective magnification. Images were adjusted for brightness and the number of LC3B positive dots was quantified using ImageJ software (1.64r; NIH, Bethesda, MD, USA), using a modified plugin as previously described [37].

\section{Patients and tissue samples}

Patient tissue of esophageal adenocarcinoma resection specimens was collected from the archive of the Institute of Pathology, University of Bern, Bern, Switzerland. The patients were consecutively treated between 1991 and 2011 at the Department of Visceral Surgery of the Inselspital, Bern, Switzerland. We selected only cases that were primarily resected without prior neoadjuvant chemo- or radiochemotherapy or adjuvant treatment. The use of human archival pathological tissue for TMA based studies was approved by the local ethics committee (Kantonale Ethikkommission Bern, Switzerland, 200/14: Identification of novel prognostic and predictive biomarkers using tissue microarrays (TMAs) constructed at the INSTITUTE of Pathology, University of Bern).

\section{Tissue microarray}

Buffered formalin fixed paraffin embedded (FFPE) tissue from primary resected EAC $(n=116$; three punches taken from tumor center and tumor periphery 
respectively), Barrett's mucosa ( $n=21)$, adjacent nonneoplastic esophageal mucosa $(n=60)$, adjacent nonneoplastic gastric mucosa $(n=68)$, EAC lymph node metastasis $(n=58)$ and EAC distant metastasis $(n=18)$ was used to construct a next generation tissue microarray (ngTMA), with digital annotation of scanned slides and automatic transferal of the punches, as previously described [38]. Three punches from each location were transferred to the TMA acceptor block from each tissue (0.6 $\mathrm{mm}$ punches).

\section{Immunohistochemical staining, scoring and subclassification}

The ngTMA was sectioned at $4 \mu \mathrm{m}$. After deparaffination, rehydration, and antigen retrieval, immunohistochemical staining was performed using an automated immunostainer (Bond RX, Leica Biosystems, Heerbrugg, Switzerland) as previously described [10]. Briefly the anti-LC3B antibody (Novus Biologicals \#NB600-1384) was diluted 1:4000 in tris buffer and incubated at $95^{\circ} \mathrm{C}$ for $30 \mathrm{~min}$. The anti-p62/SQSTM1 antibody (MBL rabbit polyclonal, \#PM045, LabForce, Nunningen, Switzerland) was diluted 1:9000 in tris buffer and incubated at $95^{\circ} \mathrm{C}$ for $30 \mathrm{~min}$. Visualization was performed using the Bond Polymer Refine Detection kit (Leica Biosystems, product number) per manufacturer's instructions. IHC stainings were scored according to a previously established protocol [10]. The immunohistochemical stainings were independently scored by two observers (OA and RL) on a Zeiss Axioskop microscope at 40x objective magnification. Discrepant results were re-evaluated at a double-header microscope.

For the purpose of further analysis IHC scores were categorized as either "low" or "high" for each staining pattern according to the prognostic impact of the single scores (for survival curves of individual scores see Supplementary Figures 2 and 3). For LC3B dot-like staining scores 0 and 1 were classified as low, while scores 2 and 3 were classified as high. The low category of p62 dot-like staining was assigned to score 0 , while scores 1 , 2 and 3 were assigned to the high category. The scores for p62 cytoplasmic staining were similarly subdivided, while score 0 for p62 nuclear staining was classified as low and scores 1 and 2 as high. Finally a p62 sum score was calculated by adding the individual scores of the dotlike, cytoplasmic and nuclear staining patterns together. The p62 sum score ranged from 0 to 6 . Subsequently the p62 sum score was also subdivided in low (score 0 and 1) and high (from score 2 to 6 ) groups.

According to work published by Iwade et al. [12] we stratified our dataset into four subtypes with respect to p62 staining patterns and subcellular location: low p62 cytoplasmic/low p62 nuclear (LL), high p62 cytoplasmic/ low p62 nuclear (HL), low p62 cytoplasmic/high p62 nuclear (LH) and high p62 cytoplasmic/high p62 nuclear $(\mathrm{HH})$. The cytoplasmic score was calculated by adding the dot-like and cytoplasmic staining scores together with scores 0 and 1 being classified as low and scores 2 through 5 being classified as high (see Supplementary Figure 3). The HL and LH categories were combination into a "mixed" group and used for subsequent analysis. According to Liu and colleagues [13] we stratified our data set according to LC3B and p62 staining patterns, resulting in three groups: low LC3B/low p62 (LL), mixed (low LC3B/high p62 or vice versa) and high LC3B/high p62. In the context of this analysis the p62 sum score low/high categories were used as previously mentioned.

Host inflammatory response to the tumors were scored according to Brown et al., who recognized three different grades; i.e. sparse, moderate and pronounced based on histomorphology by Hematoxylin \& Eosin staining [39]. For the purpose of this study this grading was simplified into low (sparse) and high (moderate and high) with an equal distribution of the two categories and estimated across all six TMA punches. Examples of low and high inflammatory response are given in Supplementary Figure 5.

\section{Statistical analysis}

The SPSS 23 software (SPSS Inc, Chicago, IL, USA) was used for descriptive and comparative statistical analysis. Associations between staining patterns and clinicopathological parameters were evaluated using simple cross tabs ( $\chi^{2}$-test or Fisher's exact test). Binded samples were evaluated using the Wilcoxon test. Survival analysis was performed using log rank test and Cox regression analysis (inclusion). The significance level was set at 0.05 .

\section{ACKNOWLEDGMENTS}

The authors thank Deborah Krauer for her excellent technical assistance. The authors acknowledge the Translational Research Unit of the Institute of Pathology, University of Bern, particularly Prof. Inti Zlobec and Dr. José Galván Hernández, for the excellent technical support on this project and advise in statistical questions. Furthermore, the authors wish to thank the Tumor Pathology 2 research group of the Experimental Pathology division of the Institute of Pathology, University of Bern. This study was supported by a grant from the Swiss Cancer League (KFS-3083-02-2013) awarded to RL.

\section{CONFLICTS OF INTEREST}

The authors have no conflicts of interest to declare. 


\section{REFERENCES}

1. Napier KJ, Scheerer M and Misra S. Esophageal cancer: A Review of epidemiology, pathogenesis, staging workup and treatment modalities. World journal of gastrointestinal oncology. 2014; 6:112-120.

2. Rubenstein JH and Shaheen NJ. Epidemiology, Diagnosis, and Management of Esophageal Adenocarcinoma. Gastroenterology. 2015; 149:302-317.e301.

3. B EF and Thorburn A. Recent insights into cell death and autophagy. The FEBS journal. 2015.

4. Galluzzi L, Pietrocola F, Bravo-San Pedro JM, Amaravadi RK, Baehrecke EH, Cecconi F, Codogno P, Debnath J, Gewirtz DA, Karantza V, Kimmelman A, Kumar S, Levine B, Maiuri MC, Martin SJ, Penninger J, et al. Autophagy in malignant transformation and cancer progression. The EMBO journal. 2015; 34:856-880.

5. Janji B, Viry E, Moussay E, Paggetti J, Arakelian T, Mgrditchian T, Messai Y, Noman MZ, Van Moer K, Hasmim M, Mami-Chouaib F, Berchem G and Chouaib S. The multifaceted role of autophagy in tumor evasion from immune surveillance. Oncotarget. 2016; doi: 10.18632/ oncotarget.7540.

6. Pan H, Chen L, Xu Y, Han W, Lou F, Fei W, Liu S, Jing $\mathrm{Z}$ and Sui X. Autophagy-associated immune responses and cancer immunotherapy. Oncotarget. 2016; doi: 10.18632/ oncotarget.6908.

7. Mandelbaum J, Rollins N, Shah P, Bowman D, Lee JY, Tayber O, Bernard H, LeRoy P, Li P, Koenig E, Brownell JE and D'Amore N. Identification of a lung cancer cell line deficient in atg7-dependent autophagy. Autophagy. 2015:0.

8. Ouyang DY, Xu LH, He XH, Zhang YT, Zeng LH, Cai JY and Ren S. Autophagy is differentially induced in prostate cancer LNCaP, DU145 and PC-3 cells via distinct splicing profiles of ATG5. Autophagy. 2013; 9:20-32.

9. Jiang P and Mizushima N. LC3- and p62-based biochemical methods for the analysis of autophagy progression in mammalian cells. Methods (San Diego, Calif). 2015; 75:1318.

10. Schlafli AM, Berezowska S, Adams O, Langer R and Tschan MP. Reliable LC3 and p62 autophagy marker detection in formalin fixed paraffin embedded human tissue by immunohistochemistry. European journal of histochemistry. 2015; 59:2481.

11. Sobin L, Gospodarowicz M and Wittekind C. (2010). TNM classification of malignant tumors. (New York: John Wiley \& Sons)

12. Iwadate $\mathrm{R}$, Inoue $\mathrm{J}$, Tsuda $\mathrm{H}$, Takano $\mathrm{M}$, Furuya $\mathrm{K}$, Hirasawa A, Aoki D and Inazawa J. High Expression of p62 Protein Is Associated with Poor Prognosis and Aggressive Phenotypes in Endometrial Cancer. The American journal of pathology. 2015; 185:2523-2533.

13. Liu JL, Chen FF, Lung J, Lo CH, Lee FH, Lu YC and Hung $\mathrm{CH}$. Prognostic significance of p62/SQSTM1 subcellular localization and LC3B in oral squamous cell carcinoma. British journal of cancer. 2014; 111:944-954.

14. Deretic V, Jiang S and Dupont N. Autophagy intersections with conventional and unconventional secretion in tissue development, remodeling and inflammation. Trends in cell biology. 2012; 22:397-406.

15. Lapaquette $\mathrm{P}$, Guzzo J, Bretillon L and Bringer MA. Cellular and Molecular Connections between Autophagy and Inflammation. Mediators of inflammation. 2015; 2015:398483.

16. O'Donovan TR, O'Sullivan GC and McKenna SL. Induction of autophagy by drug-resistant esophageal cancer cells promotes their survival and recovery following treatment with chemotherapeutics. Autophagy. 2011; 7:509524.

17. O'Donovan TR, Rajendran S, O'Reilly S, O'Sullivan GC and McKenna SL. Lithium Modulates Autophagy in Esophageal and Colorectal Cancer Cells and Enhances the Efficacy of Therapeutic Agents In Vitro and In Vivo. PloS one. 2015; 10:e134676.

18. Roesly HB, Khan MR, Chen HD, Hill KA, Narendran N, Watts GS, Chen X and Dvorak K. The decreased expression of Beclin-1 correlates with progression to esophageal adenocarcinoma: the role of deoxycholic acid. American journal of physiology Gastrointestinal and liver physiology. 2012; 302:G864-872.

19. El-Mashed S, O'Donovan TR, Kay EW, Abdallah AR, Cathcart MC, O'Sullivan J, O'Grady A, Reynolds J, O'Reilly S, O'Sullivan GC and McKenna SL. LC3B globular structures correlate with survival in esophageal adenocarcinoma. BMC cancer. 2015; 15:582.

20. Martinet W, Schrijvers DM, Timmermans JP, Bult H and De Meyer GR. Immunohistochemical analysis of macroautophagy: recommendations and limitations. Autophagy. 2013; 9:386-402.

21. He H, Dang Y, Dai F, Guo Z, Wu J, She X, Pei Y, Chen Y, Ling W, Wu C, Zhao S, Liu JO and Yu L. Post-translational modifications of three members of the human MAP1LC3 family and detection of a novel type of modification for MAP1LC3B. The Journal of biological chemistry. 2003; 278:29278-29287.

22. Wu J, Dang Y, Su W, Liu C, Ma H, Shan Y, Pei Y, Wan $\mathrm{B}$, Guo J and $\mathrm{Yu}$ L. Molecular cloning and characterization of rat LC3A and LC3B-two novel markers of autophagosome. Biochemical and biophysical research communications. 2006; 339:437-442.

23. Bao L, Chandra PK, Moroz K, Zhang X, Thung SN, Wu $\mathrm{T}$ and Dash S. Impaired autophagy response in human hepatocellular carcinoma. Experimental and molecular pathology. 2014; 96:149-154.

24. Inoue D, Suzuki T, Mitsuishi Y, Miki Y, Suzuki S, Sugawara S, Watanabe M, Sakurada A, Endo C, Uruno A, Sasano H, Nakagawa T, Satoh K, Tanaka N, Kubo $\mathrm{H}$, Motohashi $\mathrm{H}$, et al. Accumulation of p62/SQSTM1 
is associated with poor prognosis in patients with lung adenocarcinoma. Cancer science. 2012; 103:760-766.

25. Mohamed A, Ayman A, Deniece J, Wang T, Kovach C, Siddiqui MT and Cohen C. P62/Ubiquitin IHC Expression Correlated with Clinicopathologic Parameters and Outcome in Gastrointestinal Carcinomas. Frontiers in oncology. 2015; 5:70.

26. Ren F, Shu G, Liu G, Liu D, Zhou J, Yuan L and Zhou J. Knockdown of p62/sequestosome 1 attenuates autophagy and inhibits colorectal cancer cell growth. Molecular and cellular biochemistry. 2014; 385:95-102.

27. Sakakura K, Takahashi H, Kaira K, Toyoda M, Oyama $\mathrm{T}$ and Chikamatsu K. Immunological significance of the accumulation of autophagy components in oral squamous cell carcinoma. Cancer science. 2015; 106:1-8.

28. Komatsu M, Waguri S, Koike M, Sou YS, Ueno T, Hara T, Mizushima N, Iwata J, Ezaki J, Murata S, Hamazaki J, Nishito Y, Iemura S, Natsume T, Yanagawa T, Uwayama $\mathrm{J}$, et al. Homeostatic levels of p62 control cytoplasmic inclusion body formation in autophagy-deficient mice. Cell. 2007; 131:1149-1163.

29. Duran A, Linares JF, Galvez AS, Wikenheiser K, Flores JM, Diaz-Meco MT and Moscat J. The signaling adaptor p62 is an important NF-kappaB mediator in tumorigenesis. Cancer cell. 2008; 13:343-354.

30. Ling J, Kang Y, Zhao R, Xia Q, Lee DF, Chang Z, Li J, Peng B, Fleming JB, Wang H, Liu J, Lemischka IR, Hung MC and Chiao PJ. KrasG12D-induced IKK2/beta/ NF-kappaB activation by IL-1alpha and p62 feedforward loops is required for development of pancreatic ductal adenocarcinoma. Cancer cell. 2012; 21:105-120.

31. Moscat J and Diaz-Meco MT. p62: a versatile multitasker takes on cancer. Trends in biochemical sciences. 2012; 37:230-236.

32. Gao C, Cao W, Bao L, Zuo W, Xie G, Cai T, Fu W, Zhang J, Wu W, Zhang X and Chen YG. Autophagy negatively regulates Wnt signalling by promoting Dishevelled degradation. Nature cell biology. 2010; 12:781-790.
33. Kuo TC, Chen CT, Baron D, Onder TT, Loewer S, Almeida S, Weismann CM, Xu P, Houghton JM, Gao FB, Daley GQ and Doxsey S. Midbody accumulation through evasion of autophagy contributes to cellular reprogramming and tumorigenicity. Nature cell biology. 2011; 13:1214-1223.

34. Linares JF, Amanchy R, Greis K, Diaz-Meco MT and Moscat J. Phosphorylation of p62 by cdk1 controls the timely transit of cells through mitosis and tumor cell proliferation. Molecular and cellular biology. 2011; 31:105117.

35. Rello-Varona S, Lissa D, Shen S, Niso-Santano M, Senovilla L, Marino G, Vitale I, Jemaa M, Harper F, Pierron G, Castedo M and Kroemer G. Autophagic removal of micronuclei. Cell cycle (Georgetown, Tex). 2012; 11:170176.

36. Pankiv S, Lamark T, Bruun JA, Overvatn A, Bjorkoy G and Johansen T. Nucleocytoplasmic shuttling of p62/SQSTM1 and its role in recruitment of nuclear polyubiquitinated proteins to promyelocytic leukemia bodies. The Journal of biological chemistry. 2010; 285:5941-5953.

37. Dagda RK, Zhu J, Kulich SM and Chu CT. Mitochondrially localized ERK2 regulates mitophagy and autophagic cell stress: implications for Parkinson's disease. Autophagy. 2008; 4:770-782.

38. Zlobec I, Koelzer VH, Dawson H, Perren A and Lugli A. Next-generation tissue microarray (ngTMA) increases the quality of biomarker studies: an example using CD3, $\mathrm{CD} 8$, and CD45RO in the tumor microenvironment of six different solid tumor types. Journal of translational medicine. 2013; 11:104.

39. Brown M, Sillah K, Griffiths EA, Swindell R, West CM, Page RD, Welch IM and Pritchard SA. Tumour budding and a low host inflammatory response are associated with a poor prognosis in oesophageal and gastro-oesophageal junction cancers. Histopathology. 2010; 56:893-899. 